\title{
PHYSICAL STUDIES OF ASTEROIDS BY POLARIZATION OF THE LIGHT
}

\author{
AUDOUIN DOLLFUS \\ Observatoire de Paris
}

Curves of polarization are available at present for asteroids Vesta, Ceres, Pallas, Iris, Flora, and Icarus. These curves are compared with those of the satellites of Jupiter and Mercury, the Moon, and Mars. Laboratory simulations had already proved that the Moon's surface behaves like a powder of pulverized basalts; the recent confirmation by direct exploration is proving the significance of the method for remote determination of the surface properties of celestial bodies. The simulation of the Martian surface is found on small grained powders oxidized by ferreous limonite or goethite. New laboratory measurements were conducted to prepare the simulation of the asteroidal surfaces. Samples of the lunar surface returned to Earth provide impact-generated regolith and bare rocks superficially pitted and etched by impacts of the types suggested to be found on asteroidal surfaces; they were analyzed polarimetrically.

Preliminary interpretations show that Vesta departs significantly from the other asteroids and cannot be covered by frost deposits or by aggregate cosmic dusts; a regolith-type surface generated by impacts or a coating of cohesive grains is indicated.

Ceres, Pallas, and Iris are darker, and their polarizations do not suggest a pure regolithic surface, but cohesive grains or aggregates of dust are indicated.

Icarus is $10^{8}$ times smaller in mass; its polarization authorizes a fluffy, loosely aggregated dust deposit; however, a cometary model with stones embedded in ice is perhaps not ruled out on the basis of the present data.

The way in which deep-space missions near the asteroidal belt can improve these results is discussed.

\section{ANALYSIS OF THE REMOTE SURFACE CHARACTERISTICS OF THE ASTEROIDS BY POLARIZATION}

Telescopic observations permit determination of the amount of polarization $P$ of the light received from asteroids. The plot of these measurements as a function of the phase angle $\alpha$ defines the "curve of polarization" of an asteroid. This curve characterizes the mineralogic properties and structural texture of the asteroidal surface. These curves can be compared from asteroid to asteroid and with other celestial bodies, and simulated by laboratory measurements on different kinds of mineralogic samples. 


\section{POLARIZATION MEASUREMENTS AVAILABLE ON ASTEROIDS}

The first curves of polarization on asteroids were derived in France by $B$. Lyot (1934). He used a photographic polarimeter attached to the $100 \mathrm{~cm}$ reflector of Meudon Observatory. ${ }^{1}$ A curve of polarization was obtained for Vesta in 1934 and Ceres in 1935. These curves were published later by A. Dollfus (1961) and are reproduced again in figures 1 and 2. The curve for Vesta (fig. 1) starts with a negative branch (electric vector maximum in the plane through the Sun, asteroid, and Earth) having a minimum of about $P_{\min }=-1.0$ percent near $\alpha=12^{\circ}$ and then rises to cross the $P=0$ value at $\alpha=26^{\circ}$. Ceres (fig. 2 ) displays a more pronounced negative branch with $P_{\mathrm{m} \text { in }}$ near -1.3 percent and $\alpha_{0}=17^{\circ}$. Then, the polarization is positive (major electric vector perpendicular to the plane of vision) and rises steeply.

Later, S. Provin (1955), with the assistance of J. S. Hall and A. A. Hoag at the U.S. Naval Observatory, Washington, D.C., used a photoelectric polarimeter on Ceres, Pallas, and Iris. His curves were republished with additional information by A. Dollfus (1961). Figure 3 reproduces the curves for Ceres; the agreement with Lyot's curves (fig. 2) is not perfect, the negative branch being more pronounced and the slope, near the inversion point, being steeper. Pallas (fig. 4) is similar to Ceres. For Iris (fig. 5), Provin followed the variation of polarization as a function of time during more than a complete photometric light period (fig. 6); the phase angle was $27^{\circ}$ and provided an average polarization of +1.2 percent; no significant variations were detected, proving

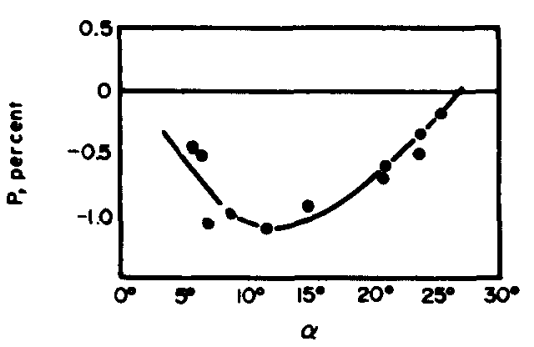

Figure 1.-Curve of polarization of Vesta. (1934 observations of B. Lyot; $100 \mathrm{~cm}$ refractor of Meudon Observatory; photographic polarimeter.)

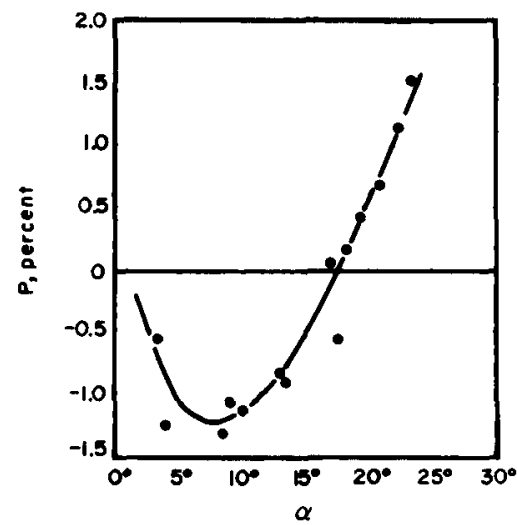

Figure 2.-Curve of polarization of Ceres. (1935 observations of B. Lyot; $100 \mathrm{~cm}$ reflector of Meudon Observatory; photographic polarimeter.)

\footnotetext{
${ }^{1}$ See p. 91 .
} 


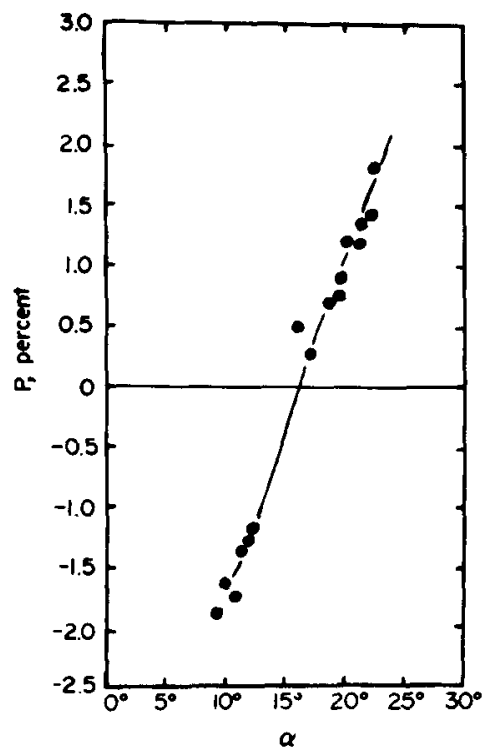

Figure 3.-Curve of polarization of Ceres. (1954 observations of D. C. Provin, U.S. Naval Observatory; photoelectric polarimeter.)
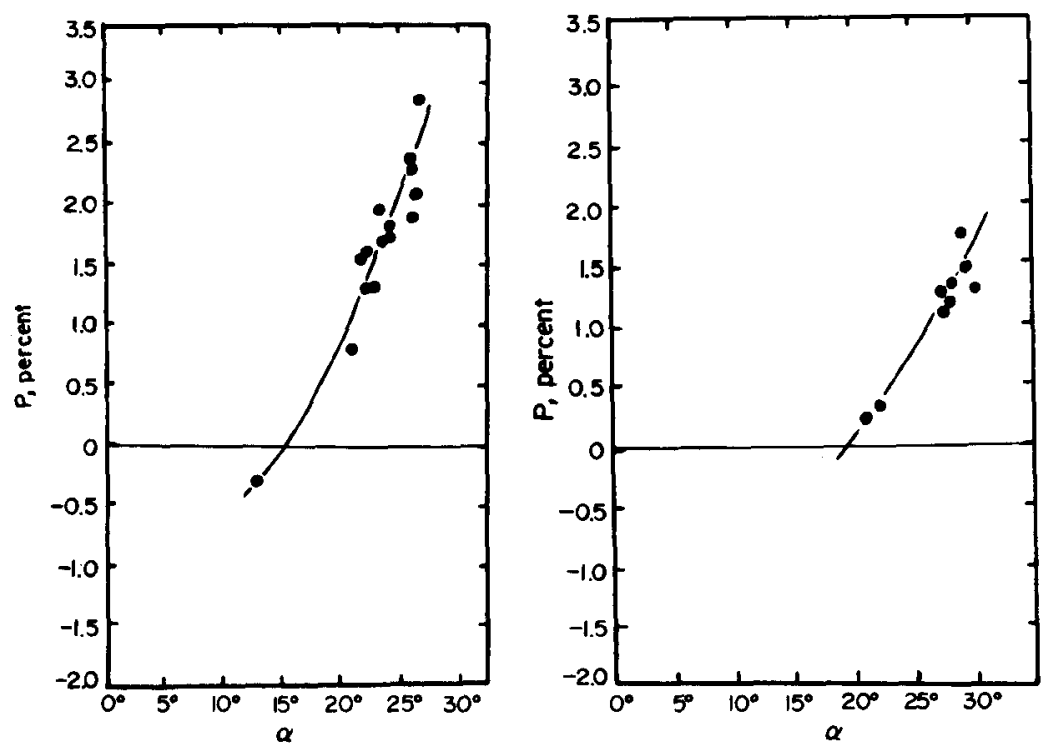

Figure 4.-Curve of polarization of Pallas. Figure 5.-Curve of polarization of Iris. (Observations of D. C. Provin, U.S. Naval Observatory; photoelectric polarimeter.)

(Observations of D. C. Provin, U.S. Naval Observatory; photoelectric polarimeter.) 


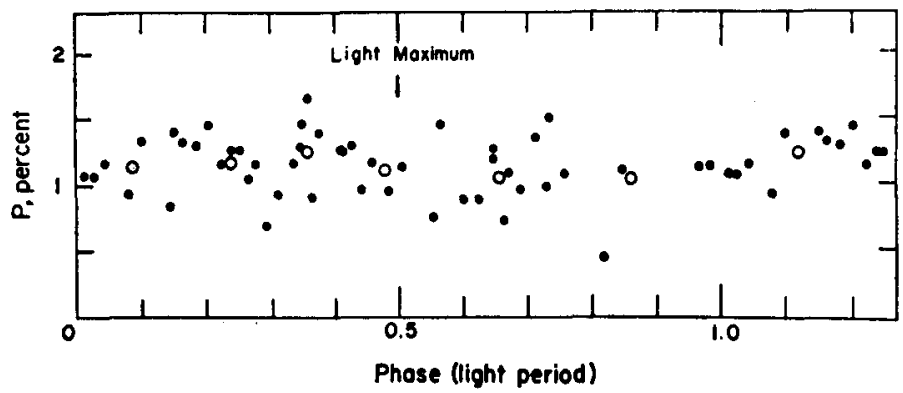

Figure 6. -Sixty-seven measurements of the amount of polarization of lris, near $\alpha=27^{\circ}$, plotted as a function of the phase of the lightcurve. Open circles give normal points. (Observations of D. C. Provin.)

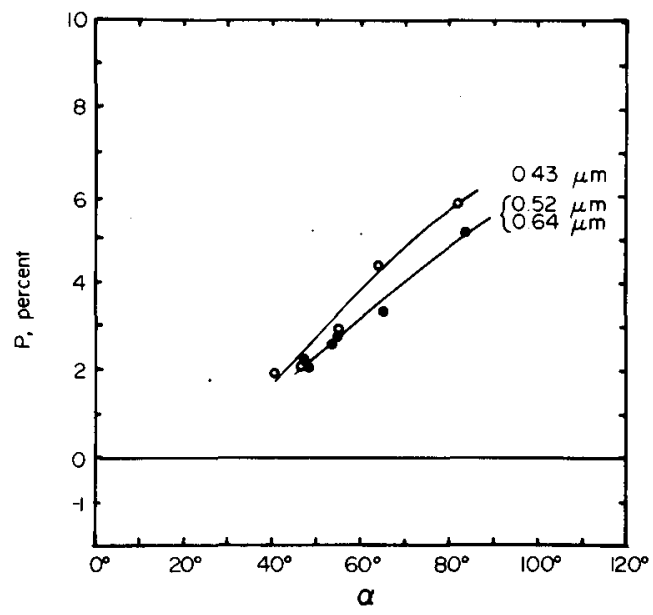

Figure 7.-Curves of polarization of Icarus obtained on the occasion of close encounter with Earth in 1968 by T. Gehrels. The values for 0.64 and $0.52 \mu \mathrm{m}$ are grouped in the same curve; the other curve and open circles are for $0.43 \mu \mathrm{m}$.

that the physical properties of the surface were the same on the different parts of the asteroid successively seen from Earth as a result of its rotation. ${ }^{2}$

The next polarization curve for an asteroid was obtained by T. Gehrels et al. (1970) on Icarus. Making use of its flyby in the vicinity of Earth in 1968, the authors collected photoelectric polarization measurements in seven colors at the Catalina Station, Arizona. Figure 7 shows the polarization curves in red and blue light derived from the published measurements. Figure 8, taken from the Gehrels publication, compares the polarization (normalized) of Icarus, as a

${ }^{2}$ See, however, p. 72 of Applied Optics 2, 1963; Provin may have been observing Iris near pole-on aspect. 


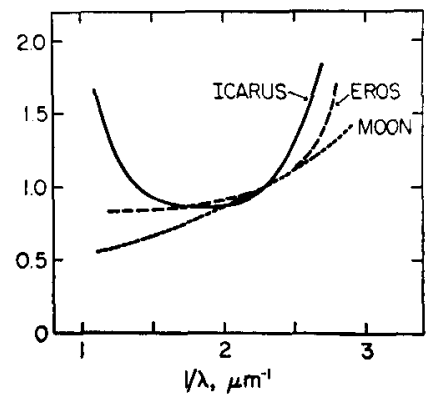

Figure 8.-Normalized polarizations of Icarus, Eros, and the Moon as functions of the inverse of wavelength. (Measurements for Eros are unpublished; observations of B. H. Zellner.)

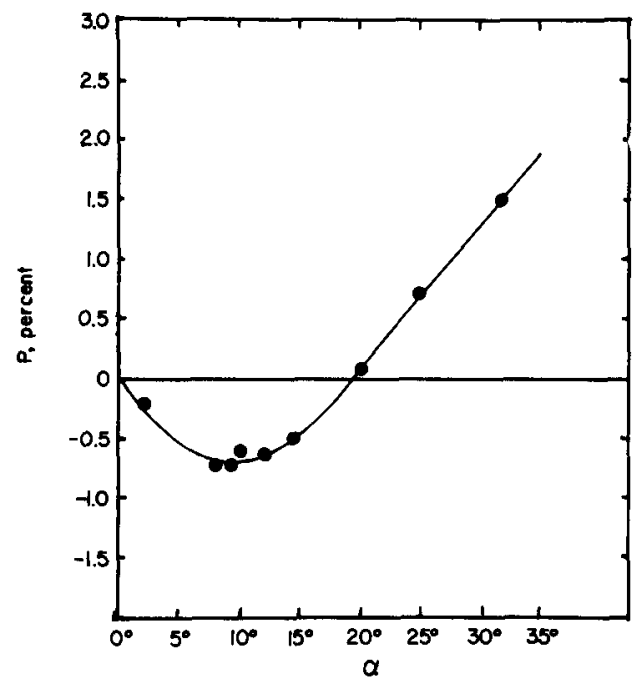

Figure 9.-Curve of polarization of Flora obtained in 1968-69 by J. Veverka at Harvard Observatory. (Photoelectric polarimeter.)

function of the inverse of the wavelength, with Mare Crisium of the Moon and unpublished results on Eros.

In 1968-69, J. Veverka (1971), using a photoelectric polarimeter with the Harvard $155 \mathrm{~cm}$ telescope, derived the polarization curve for Flora reproduced in figure 9.

\section{COMPARISON WITH OTHER CELESTIAL OBJECTS}

It is relevant to compare these polarization curves of Ceres, Pallas, Vesta, Iris, Icarus, and Flora with those of other celestial bodies practically devoid of atmospheres. 
Figure 10 shows the polarization curves of the four major satellites of Jupiter obtained by A. Dollfus (unpublished) with a photoelectric polarimeter attached to the $60 \mathrm{~cm}$ Meudon reflector and the $107 \mathrm{~cm}$ Pic-du-Midi reflector. The negative branches are systematically less pronounced, and the inversion angle smaller, than for asteroids.
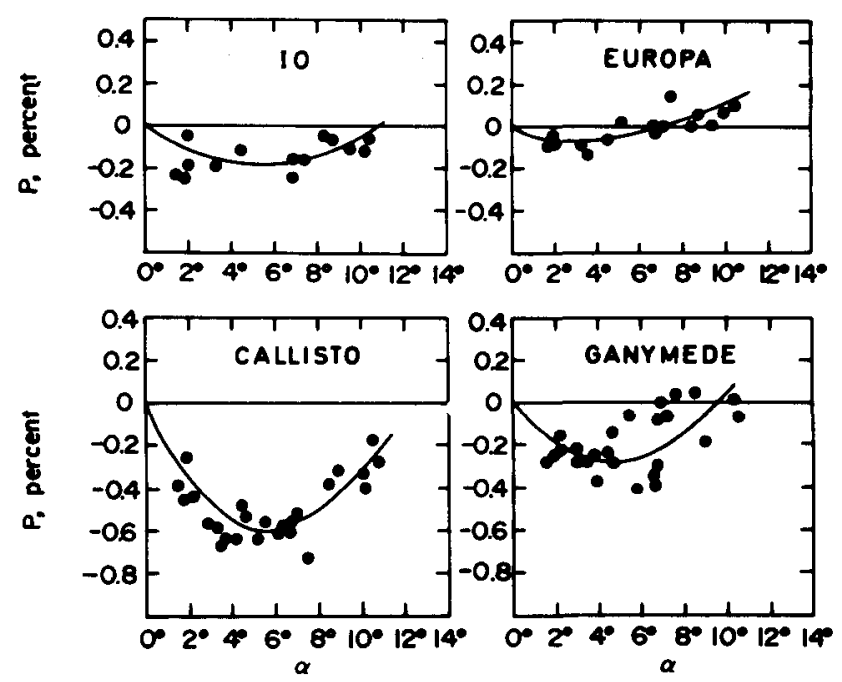

Figure 10.-Curves of polarization of the four Galilean satellites of Jupiter obtained by A. Dollfus with the $60 \mathrm{~cm}$ reflector of Meudon Observatory and the $107 \mathrm{~cm}$ reflector of Pic-du-Midi Observatory. (Photoelectric polarimeter; previously unpublished results.)

Figure 11 shows the complete polarization curve in orange light $(580 \mathrm{~nm})$ for Mercury based on observations by B. Lyot (1929) and A. Dollfus (unpublished). The inversion angle occurred at $24^{\circ}$ and the negative branch is well pronounced.

Figure 12 shows in great detail the negative branch for the polarization curve of the Moon. This curve is almost identical to the case of Mercury; the minimum of polarization occurs near $\alpha=12^{\circ}$ with the value $P_{\min }=-1.2$ percent. The inversion angle occurs at $\alpha_{0}=24^{\circ}$ and is almost independent of the albedo and the area on the Moon. Then, the polarization is positive and the steepness of the curve increases as albedo and wavelength decrease.

Figure 13 illustrates the polarization curves of planet Mars at three wavelengths. The observations, collected by A. Dollfus and J. Focas (1969), only include measurements selected when the atmosphere of Mars was apparently free of aerosols. The negative branch for $\lambda=0.61 \mu \mathrm{m}$ is almost identical to the case of the Moon and Mercury. 


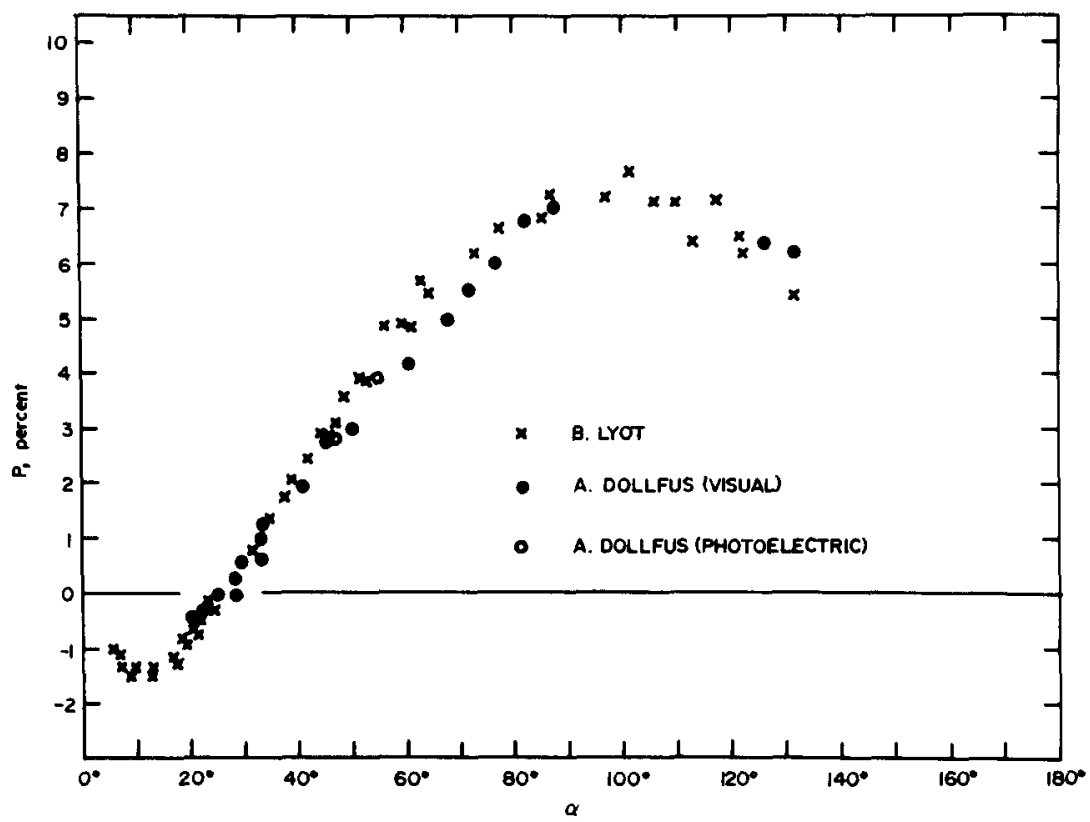

Figure 11.-Curve of polarization of planet Mercury in orange light $(580 \mathrm{~nm})$. Measurements by B. Lyot and A. Dollfus of Meudon and Pic-du-Midi Observatories. (Visual and photoelectric polarimeters.)

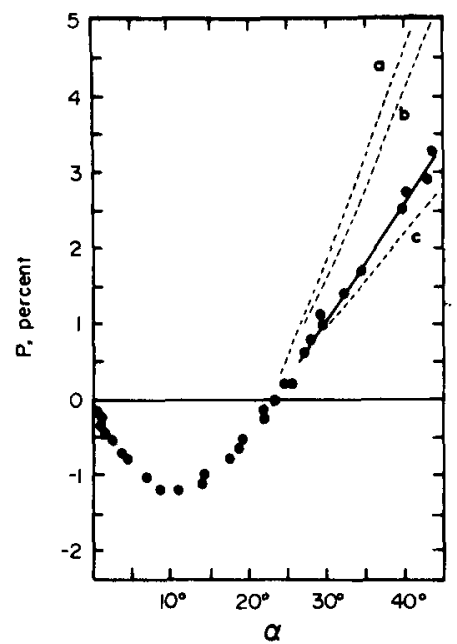

Figure 12.-Detailed observations of the negative branch of the polarization curve for the Moon (orange light). The negative branch is independent of wavelength and area on the Moon. The dashed curves $a, b$, and $c$ are from Oceanus Procellarum, Mare Serenitatis, and Hipparchus, respectively. 


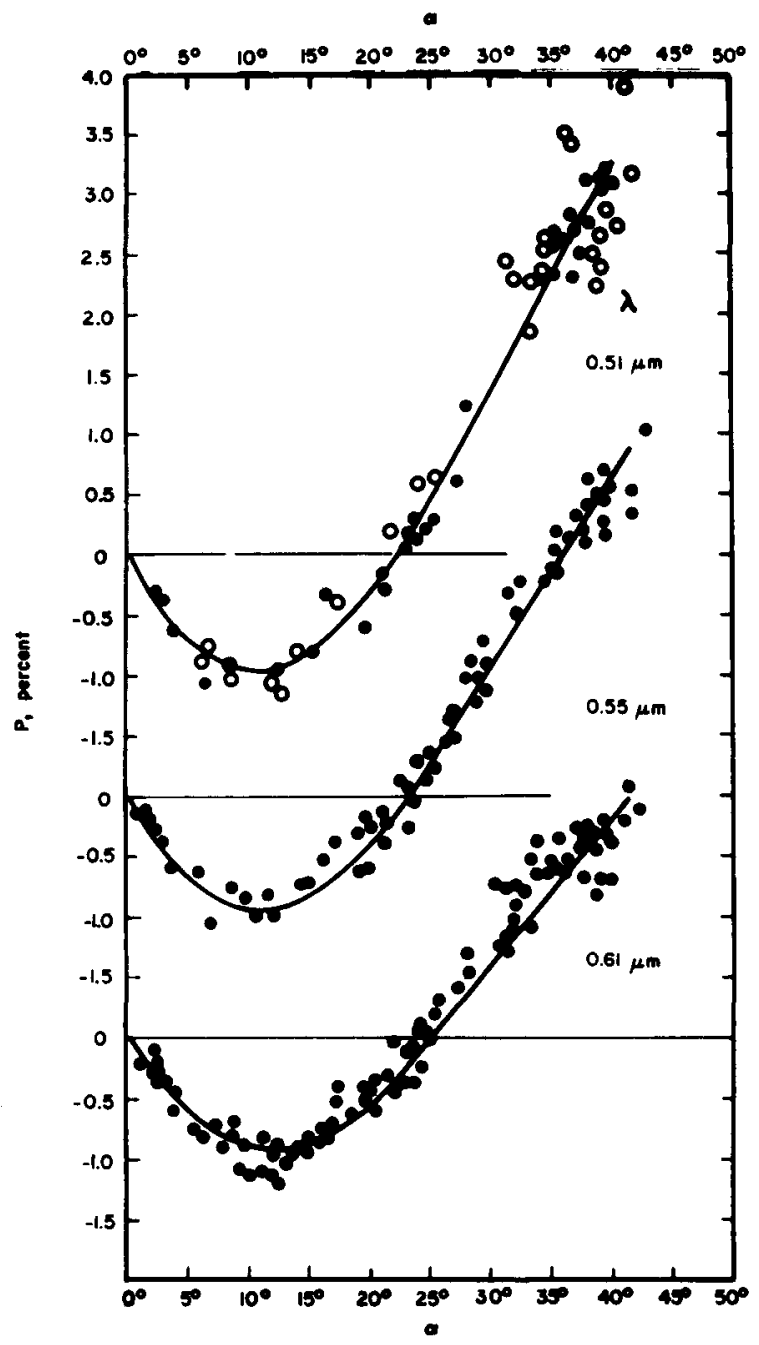

Figure 13.-Curves of polarization of the light area at the center of Mars' disk for wavelengths of $0.61,0.55$, and $0.51 \mu \mathrm{m}$. Observations by J. Focas and A. Dollfus at Meudon and Pic-du-Midi Observatories. The measurements are selected for absence of detectable aerosols in the Martian atmosphere. $\bullet$ : visual polarimetry (clear regions); $\circ$ : photoelectric polarimetry.

\section{INTERPRETATION OF THE POLARIZATION CURVES OF THE MOON, MARS, AND MERCURY}

The negative branches of the polarization curves on the Moon, Mercury, and Mars are strikingly identical, when compared to those of other celestial bodies; they are very well developed with a minimum near -1.2 percent and an 
inversion angle of about $24^{\circ}$. These properties correspond to an extreme case reached only by very specific types of materials; these criteria fortunately provide a discriminative identification of the nature and structure of these planetary surfaces.

The behavior of these negative branches was found by B. Lyot (1929) to be reproduced on volcanic ashes. Since this pioneering work, extensive studies were developed by A. Dollfus $(1955,1956,1962)$, who found this characteristic shape of the curve to be typical of fine powders mixed together and made of very absorbing material of different grain sizes as small as a few micrometers. Laboratory measurements (Dollfus, 1956) proved that multiple scattering between the absorbing grains is responsible for the negative branch.

Other optical criteria, like the spectral variation of the albedo (for Mars), or the spectral variation of the polarization maximum (for the Moon), enable one to discriminate the nature of the absorbing material from which the powder originates. For the Moon, the best optical laboratory simulation was found in finely pulverized basalts (Dollfus et al., 1971a). For the planet Mars, the small grains should be ferreous oxides, like goethite or limonite, or coatings by these oxides (Dollfus, 1956; Dollfus et al., 1969).

The remote identification was recently successfully confirmed, in the case of the Moon, by the lunar samples returned to Earth by the Apollo missions. Figure 14(a) displays a microphotograph of a pulverized basalt selected in 1954 as being the best simulation of the lunar surface for the optical properties concerned. This picture was published $16 \mathrm{yr}$ ago and again in 1962 by $\mathrm{A}$. Dollfus $(1955,1962)$. Figure $14(b)$ is a microphotograph of a sample of lunar

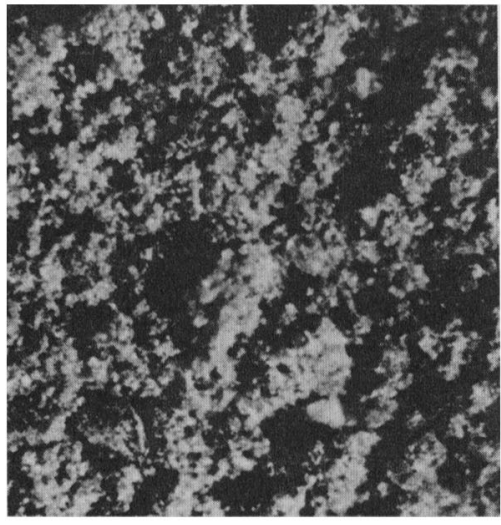

(a)

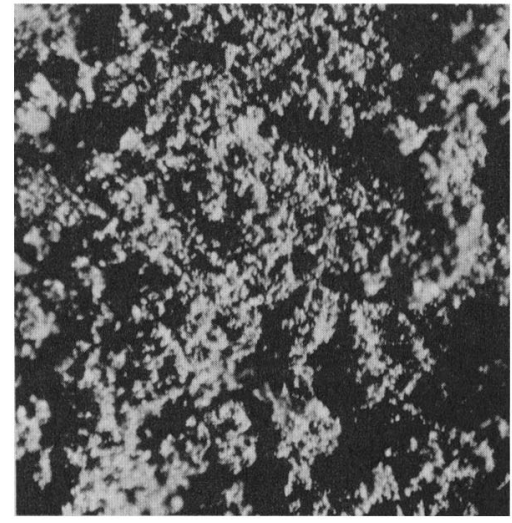

(b)

Figure 14. $-(a)$ Microphotograph of a pulverized basalt from lava flow simulating the optical properties of the lunar surface. Original size: $3 \mathrm{by} 3 \mathrm{~mm}$. This picture was obtained in 1954 and published in 1955 (Dollfus, 1955). (b) Microphotograph of an Apollo lunar fines sample obtained in 1970 in the same conditions as (a). 
fines returned to Earth by the Apollo 11 mission and photographed under the same conditions as figure $14(a)$. The striking similarity is convincing as regards the validity of the polarimetric criteria from remote identifications. (See also Geake et al., 1970.)

\section{LABORATORY POLARIZATION STUDIES RELEVANT TO ASTEROID PROBLEMS}

For the purpose of interpreting the asteroid's polarization curves, we are developing, at Meudon Observatory, a program of laboratory measurements on samples expected to simulate the asteroidal conditions. This survey is still in progress.

Among the likely candidates for the simulations of the superficial properties of asteroids are frost deposits, aggregated cosmic dust, impact-generated regolith from lunar or meteoritic material, and bare rocks superficially pitted and etched by impacts and possibly coated by adhesive grains.

Laboratory measurements on the polarization of frost deposits were obtained by A. Dollfus (1955); the amount of polarization is very low for all phase angles.

Optical measurements on deposited cosmic dust are difficult, although some preliminary indications are obtained (see below).

Particularly relevant to the asteroidal problems are the lunar surface samples returned to Earth by the Apollo missions. These minerals were exposed to the etching, weathering, or disaggregation by long space exposure and meteoritic impacts; these processes should reproduce those operating on asteroidal surfaces.

Of special interest are the samples of lunar fines collected at the surface of the regolith layer and generated by impact pulverization of the lunar surface. Figure 15 shows the polarization curves for Apollo lunar fines sample 10084,6 from Mare Tranquillitatis. The albedo in orange light is 0.075 . The curves of polarization for the full range of phase angles are given in five wavelengths. The negative branches of the polarization curves, given in figure 15, bottom right, are identical to those of the lunar surface measured through telescopes, proving that the physical structure of the powder in the laboratory retains the original configuration it had on the lunar surface. Electron microscope scanning images of the grains are given in figure 16, up to a scale releasing details smaller than the wavelength. These documents were obtained at Manchester by Dr. J. E. Geake. The texture is very complex with grains of all sizes, showing many shock features. It is the multiple scattering between all these grains that is responsible for the deep negative branch of polarization with inversion angle as high as $24^{\circ}$.

The lunar rocks should also be compared to asteroids because, as a result of the low value of their escape velocities, the small asteroids hardly retain the powder ejected by impacts (although cohesion and adherence due to vacuum 


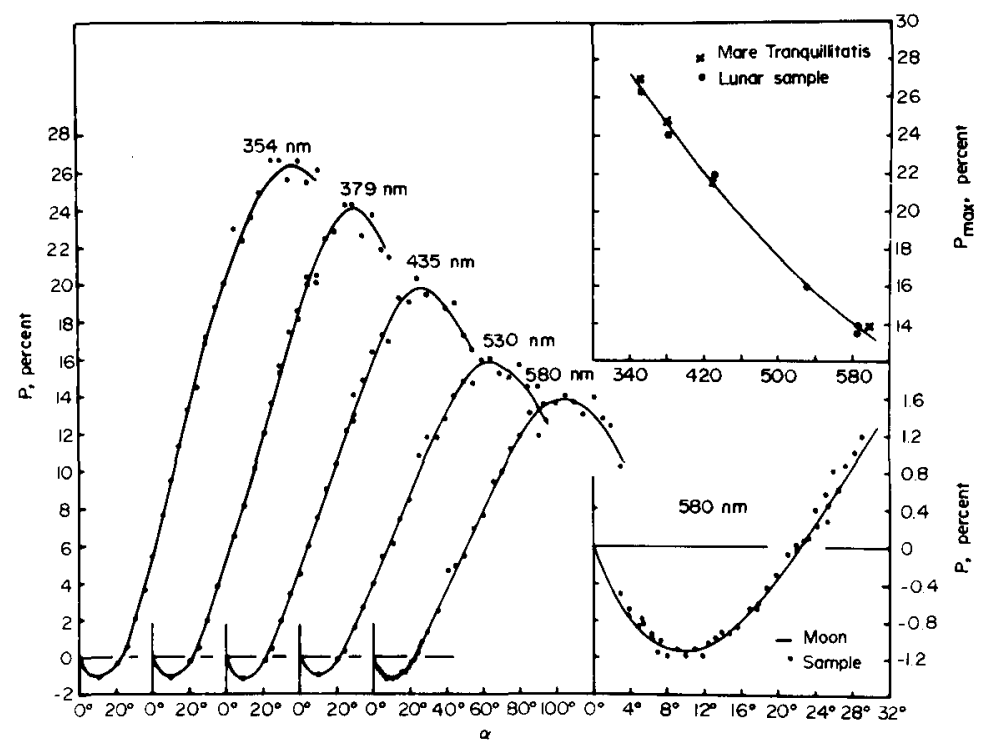

Figure 15.-Curves of polarization of the Apollo 11 lunar fines sample 10084,6 from the regolith surface of Mare Tranquillitatis. Photoelectric measurements are given in five wavelengths. (Meudon Observatory.)

may replace the gravitational retention); this is precisely the case for the rocks collected at the surface of the lunar regolith.

Figures 17 and 18 correspond to a lunar crystalline rock, Apollo sample 12051,51 . The albedo in orange light is 0.24 and the color is reddish; further information is found in Dollfus et al. (1971a). The maximum of polarization (fig. 17) is higher than on the fines (fig. 15) and occurs for larger phase angles. The negative branch, reproduced on a larger scale in figure 18 , is definitely less pronounced than for the lunar fines (fig. 16), with a minimum of -0.4 percent only (against -1.2 percent for the fines) and an inversion angle of $16^{\circ}$ (against $24^{\circ}$ for the fines). The explanation is given by the electron scanning microscope images obtained for the purpose by Dr. J. E. Geake and reproduced in figure 18; the texture is definitely smoother than the samples of fines. The picture at top right, with the highest magnification, characterizes the average texture and can be compared with the picture of the lunar fines taken with the same magnification; the multiple scattering is less efficient in these structures; furthermore, an appreciable fraction of the surface releases glassy aspects due to splashes of melted silicates reproduced in the picture at center; these areas are almost devoid of appreciable multiple scattering. Finally, because of the smaller effect of the multiple scattering, this typical lunar crystalline rock gives a small negative branch of polarization.

Figure 19 characterizes a particularly glassy lunar rock, Apollo sample 12002,102 . The albedo is 0.13 in orange light. The largest fraction of the 

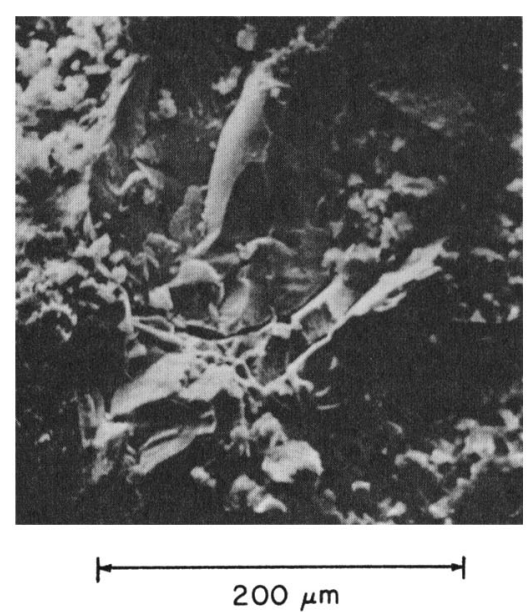
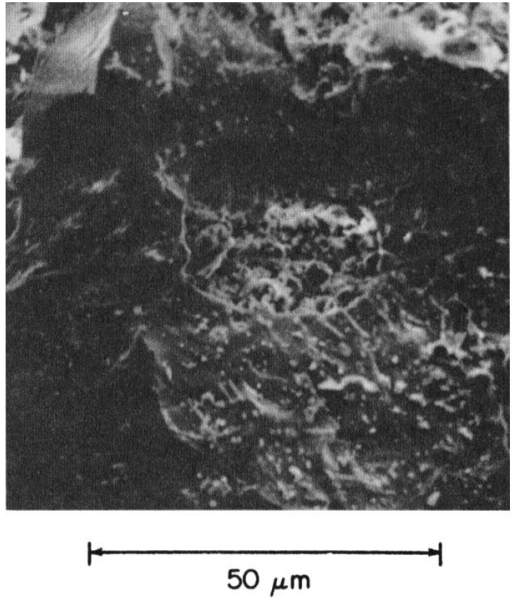
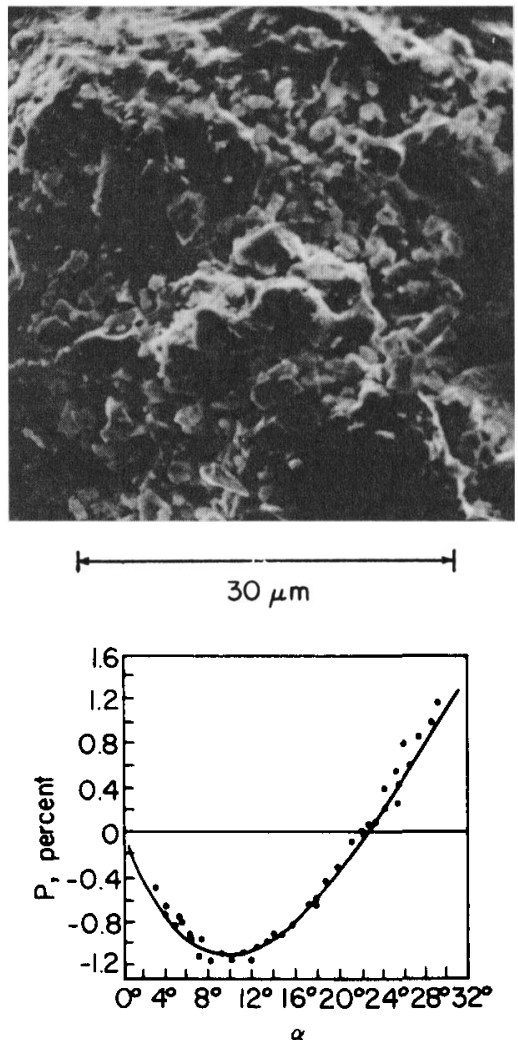

Figure 16.-Electron scanning microscope pictures of Apollo lunar fines sample 10084,6 and its negative branch of polarization. (The pictures were obtained by J. E. Geake at Manchester.) 


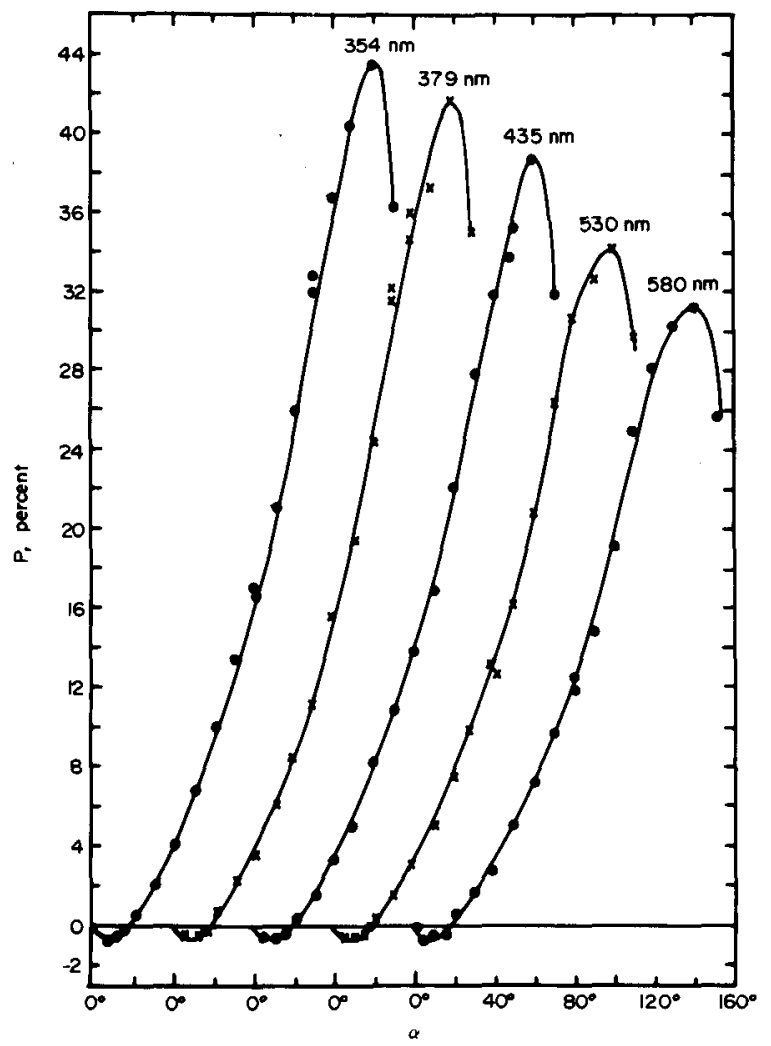

Figure 17.-Curves of polarization of Apollo lunar crystalline rock 12051,51. The photoelectric measurements were made in five wavelengths. (Meudon Observatory.)

surface appears to be almost smooth, as strikingly seen in the image on top right. A still greater enlargement is shown in the picture at center. The multiple scattering should be limited and the negative branch of polarization very small; the curve, reproduced at bottom, has a minimum of -0.2 percent only and an inversion angle of $10^{\circ}$.

Figure 20 belongs to a lunar breccia, Apollo sample 10059,36, with albedo 0.095 and an almost gray color. Each of the three images is centered on the same area, with increased enlargement, by factors of about 3 . This is a cohesive mixture of grains, exceptionally rough in all scales; some glassy grains are incorporated; the picture at center shows a glassy fragment cemented (in the upper half) with a clump of very small cohesive grains (lower half). But the multiple scattering is dominant, and the negative branch of polarization is almost as pronounced as in the case of the lunar fines.

Additional results on polarization properties of lunar rocks and fines are found in the two papers by Geake et al. (1970) and Dollfus et al. (1971b). 

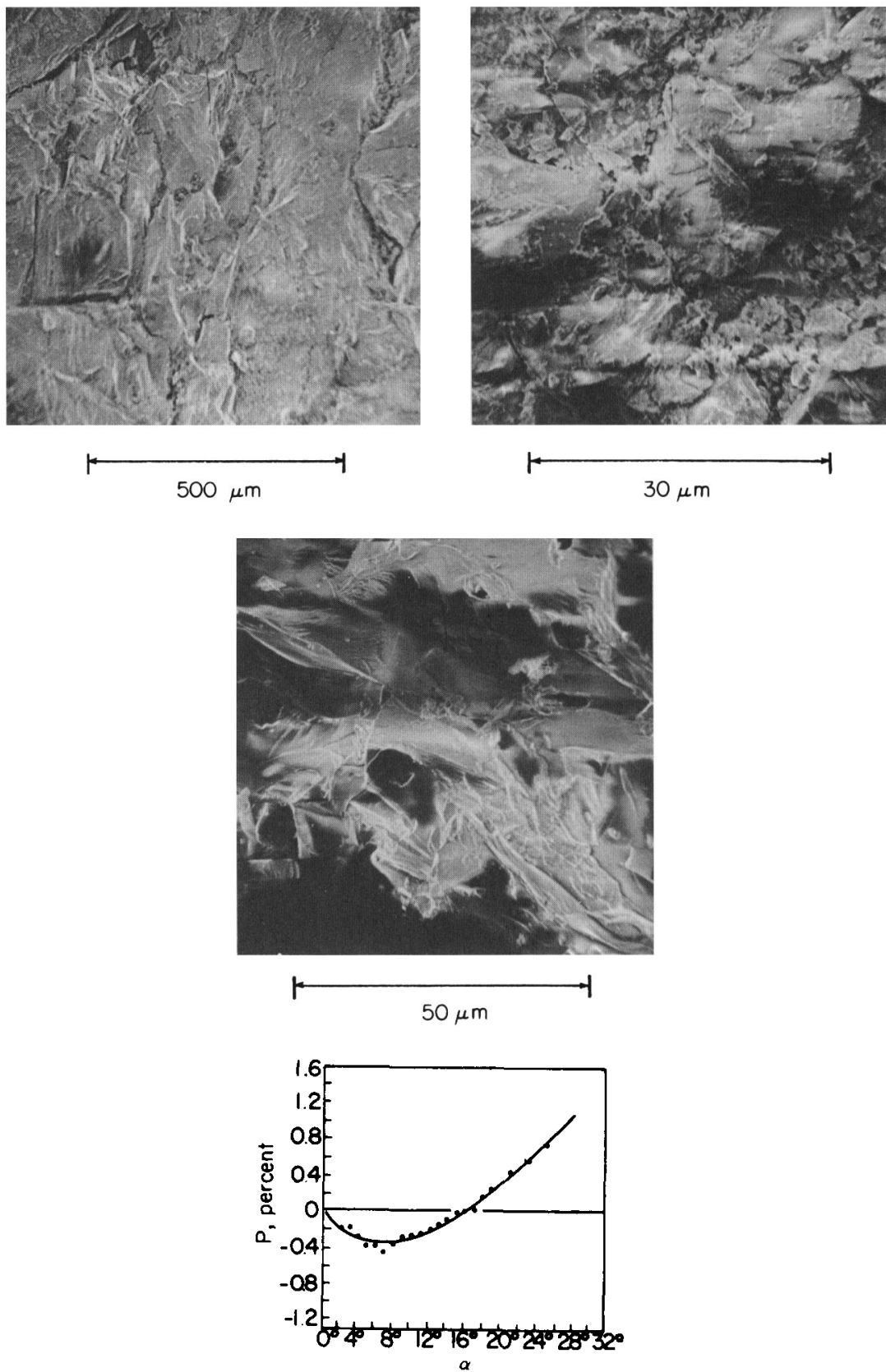

Figure 18.-Electron scanning microscope pictures of Apollo lunar crystalline rock 12051,51 and the negative branch of its polarization curve. (The pictures were obtained by J. E. Geake at Manchester.) 

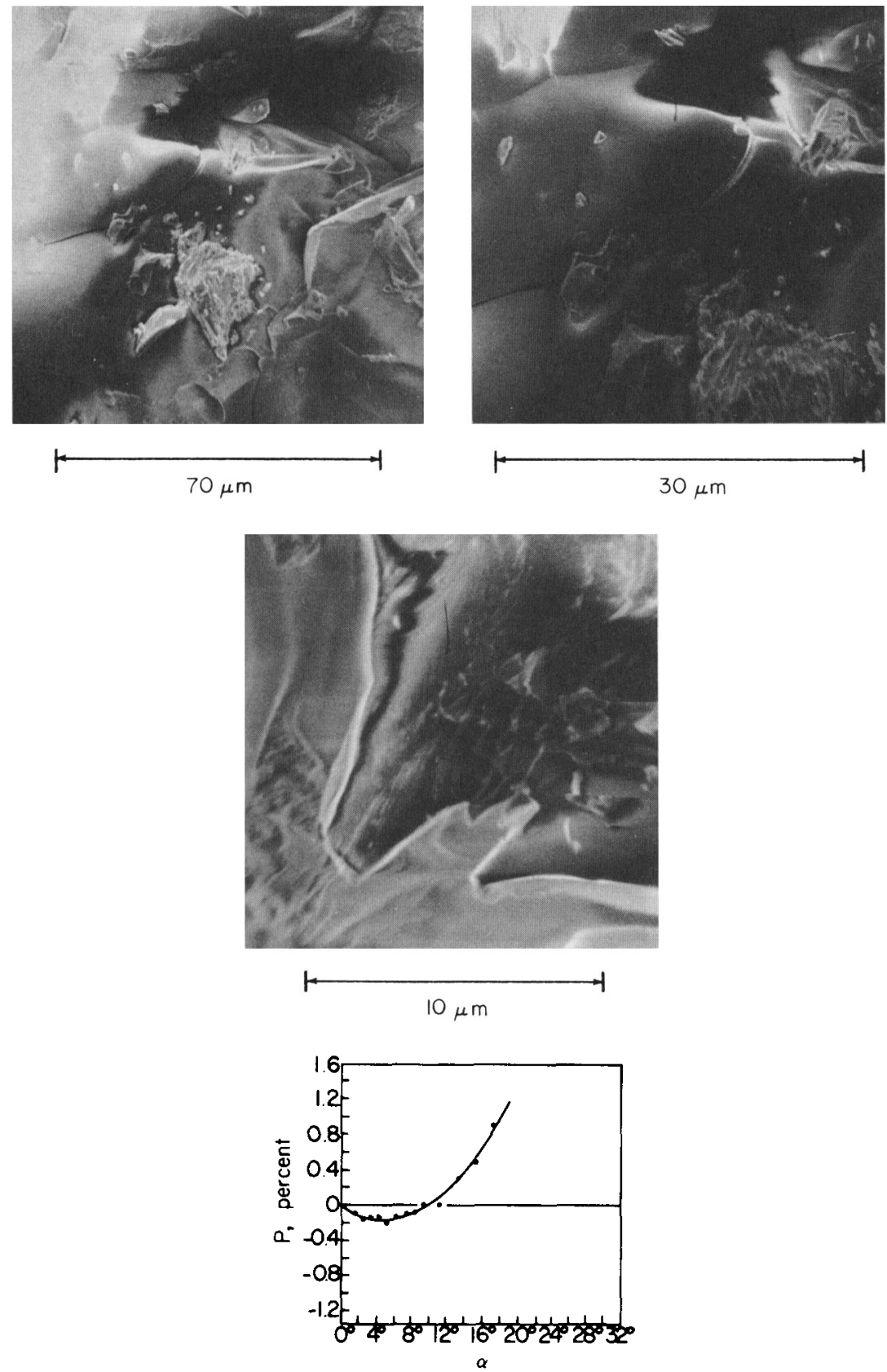

Figure 19.-Electron scanning microscope pictures of glassy A pollo lunar rock 12002,102 and the negative branch of its polarization curve. (The pictures were obtained by J. E. Geake at Manchester.) 

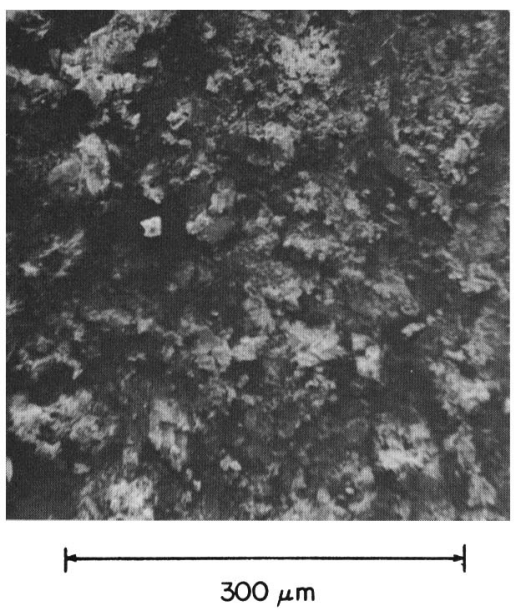
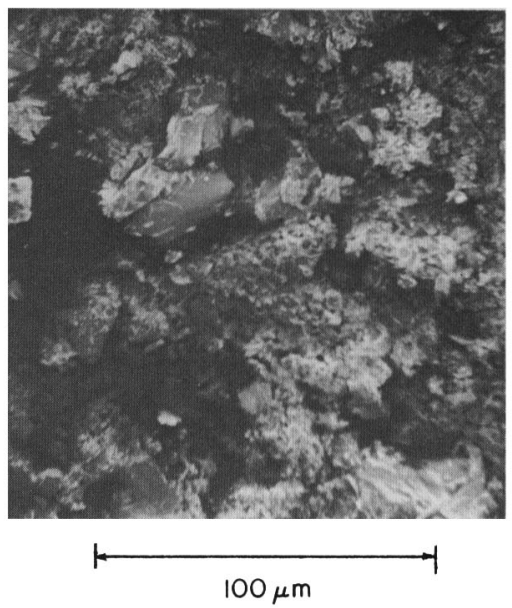
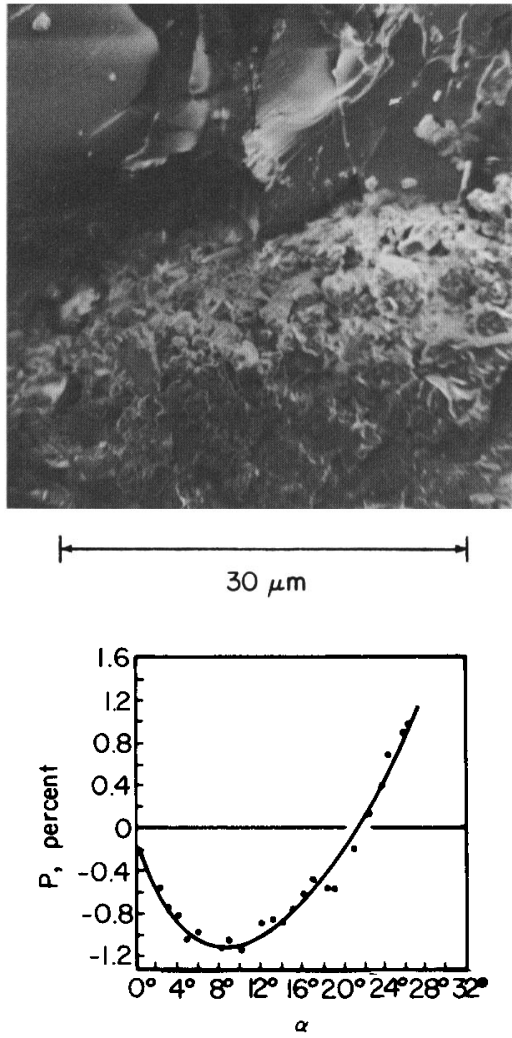

Figure 20.-Electron scanning microscope pictures of Apollo lunar breccia 10059,36 and the negative branch of its polarization curve. (The pictures were obtained by J. E. Geake at Manchester.) 


\section{INTERPRETATION OF THE POLARIZATION CURVES OF ASTEROIDS}

From the previous polarimetric results, combined with other optical or physical data available, models of the nature of the asteroidal surfaces can be derived, or at least indicated.

For Vesta the diameter is known to be of the order of $410 \mathrm{~km}$ (see A. Dollfus $^{3}$ ) and the corresponding albedo is 0.40 . This high value is compatible with frost deposits but excludes aggregated cosmic-dust coatings. The presence of polarization, in turn, excludes frost deposits, but agrees with solid surfaces.

The spectral reflectivity curves between 0.35 and $1.1 \mu \mathrm{m}$ observed by McCord et al. (1970) ${ }^{4}$ show a pronounced dip at $0.9 \mu \mathrm{m}$ found on $\mathrm{Mg}$-rich orthopyroxene and recognized on samples of basaltic achondrites. On account of its deeper negative branch, the shape of the polarization curve excludes a glassy surface of the type displayed in figure 19, and also the average lunar rock structures shown in figure 18; more multiple scattering is needed and involves more rugged surfaces.

The negative branch of polarization is not incompatible with a regolith layer resulting from fragments generated by impacts on a light basaltic achondrite mineral. For an assumed density of $3.3 \mathrm{~g} / \mathrm{cm}^{3}$, the escape velocity is $140 \mathrm{~m} / \mathrm{s}$ and a large fraction of the ejecta produced by impacts should be lost in space; however, the cohesion of grains in vacuum may help to retain sticky grains.

For Ceres, the diameter of about $770 \mathrm{~km}$ is definitely larger than that for Vesta, and the darker albedo of 0.13 is similar to the case for the Moon and Mercury. For Pallas, some inconsistencies remain between diameter measurements (see A. Dollfus ${ }^{5}$ ), but the size is intermediate between that of Ceres and Vesta, with an albedo not higher than that of Ceres. The diameter of Iris has not yet been measured but belongs to the Ceres' or Pallas' range of sizes. Although polarimetric measurements should be continued, all three of these asteroids apparently display the same type of polarization curves, with an inversion angle near $18^{\circ}$ and a negative minimum as high as 1.7 percent.

The low albedo and high negative branch of polarization exclude frost deposits on these three objects but characterize surface structures and composition definitely different from that for Vesta. The reflection spectrum from Pallas, and probably Ceres, obtained by McCord et al. (1970) does not show the $0.9 \mu \mathrm{m}$ band seen on Vesta. With escape velocities of the order of $250 \mathrm{~m} / \mathrm{s}$, these bodies should retain more easily ejected fragments from impacts, as Vesta does, but nevertheless the polarization curves depart from the characteristics of the lunar fines and, despite the similarity in albedo, exclude a pure lunar type regolithic powdered layer.

The polarimetric properties may indicate cohesive grains but do not rule out, with the present data, a loose aggregate of gently deposited cosmic dust; more observational and laboratory work is to be performed.

\footnotetext{
${ }^{3}$ See p. 28.

${ }^{4}$ See also p. 60.

${ }^{5}$ See p. 25.
} 
For Icarus, the measurements of figure 7 refer to a body belonging to a completely different range of sizes. The diameter of nearly $1 \mathrm{~km}$ (Gehrels et al., 1970) gives a mass on the order of $10^{8}$ times smaller than for the other asteroids polarimetrically analyzed. The escape velocity of $0.35 \mathrm{~m} / \mathrm{s}$ rules out the retention of any kind of ejecta resulting from impacts. The maximum of polarization of 7 percent almost excludes bare rock with an albedo of 0.26 assumed by Gehrels et al. (1970), but authorizes a fluffy, loosely aggregated deposit of (cosmic) dust. However, a cometary model with stones embedded on ice is perhaps not ruled out on the basis of the current polarimetric data available.

\section{POSSIBILITIES FOR FUTURE WORK}

In addition to the refinement and extension of the ground-based telescopic techniques currently used, major results could be expected from space missions near the asteroidal belt.

For the minor planets of the main belt, the phase angle observable from Earth is limited to the range between $0^{\circ}$ and about $30^{\circ}$. Space missions will reveal the shape of the curves near their maxima, occurring between $90^{\circ}$ and $120^{\circ}$, and determine the highest value of the polarization $P_{\max }$. Together with the albedo $A$, deduced from the direct measurements of the diameters, these values of $P_{\max }$ are basic for the telescopic determinations of the composition of these celestial bodies.

For instance, in the case of the Moon, the determinations of $P_{\max }$ for areas of different albedo plotted on a logarithmic scale as a function of $A$ display a linear relation between $\log A$ and $\log P_{\max }$, as seen in figure 21 (Dollfus and

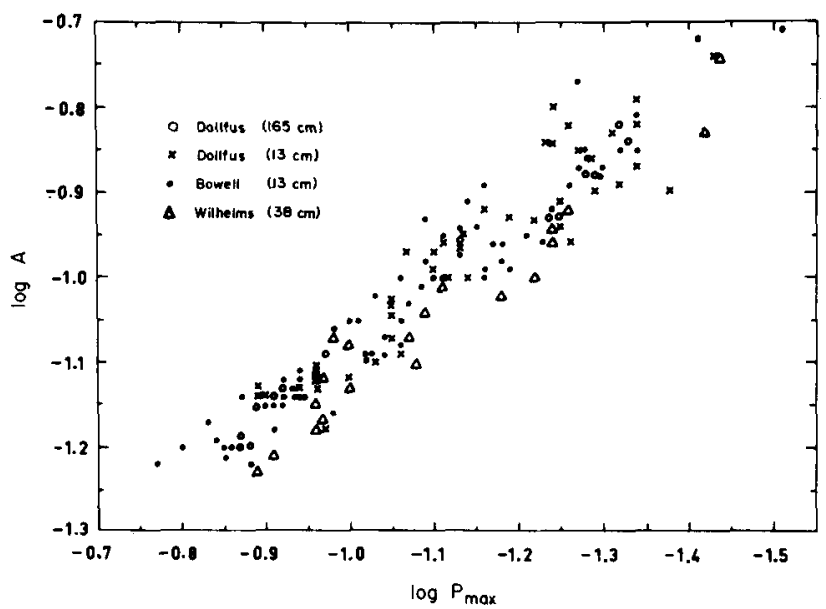

Figure 21.-Albedo of 146 areas of the lunar surface plotted as a function of their maximum of polarization $P_{\text {max }}$ in logarithmic scale. Measurements in orange light. 
Bowell, 1971). Comparisons with mineralogic samples, summarized in figure 22, demonstrate that several compositions have to be ruled out as candidates for simulation of the optical properties of the Moon, namely, sands, clays, chalks, crushed rocks, pulverized meteorites, ignimbrites, vitric basalts, and most of the volcanic ashes. On the contrary, the pulverized basalts from lava flows fit the optical properties of the lunar regolith very well (Dollfus et al., 1971a; Dollfus and Titulaer, 1971). The subsequent confirmation of this result by the direct exploration of the Moon again accredits the significance of the polarimetric technique for remote analysis of the composition of planetary surfaces.

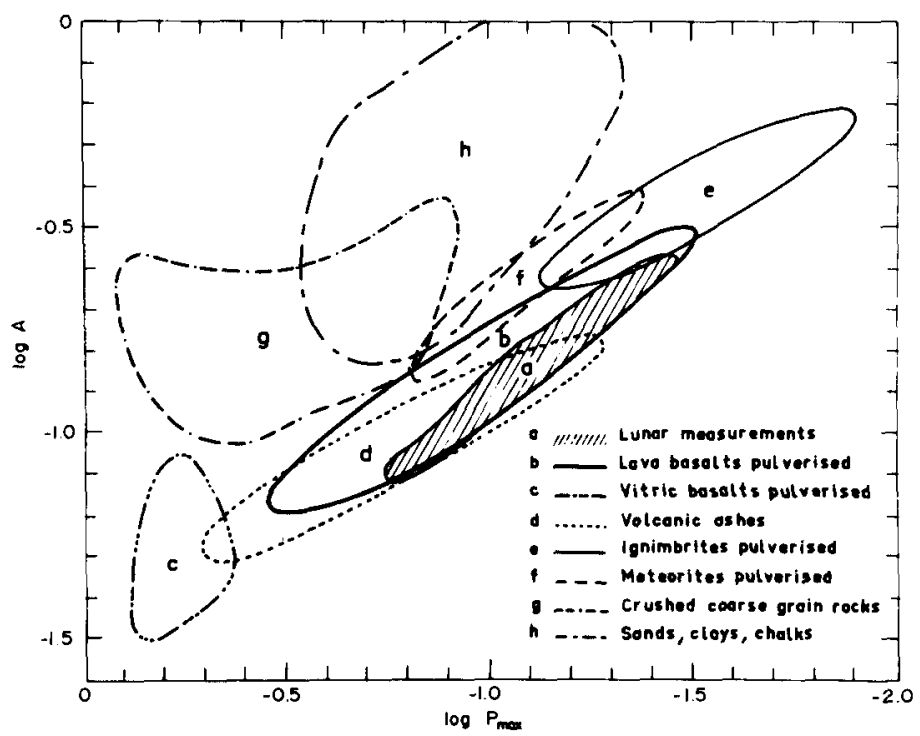

Figure 22.-Domains occupied by different kinds of terrestrial and meteoritic samples in a $\log A$ versus $\log P_{\max }$ plot similar to figure 21 . The domain for lunar measurements is derived from figure 21 after normalization of the albedo scale with the Apollo lunar samples returned to Earth.

Figure 23 further refines the determination by taking into account the spectral variation of $P_{\max }$ and $A$. Measurements at five wavelengths for each sample gave five dots in the $\log A-\log P_{\max }$ plot, alined along a segment represented in figure 23; location, length, and slope of these segments characterize still more precisely the surface and again strengthen the optical similarities between the lunar regolith and terrestrial pulverized basalts.

Spaceborne measurements on minor planets will characterize the asteroidal surfaces by dots in figure 22 and segments in figure 23 and will provide specific data for compositional comparison and simulation. 


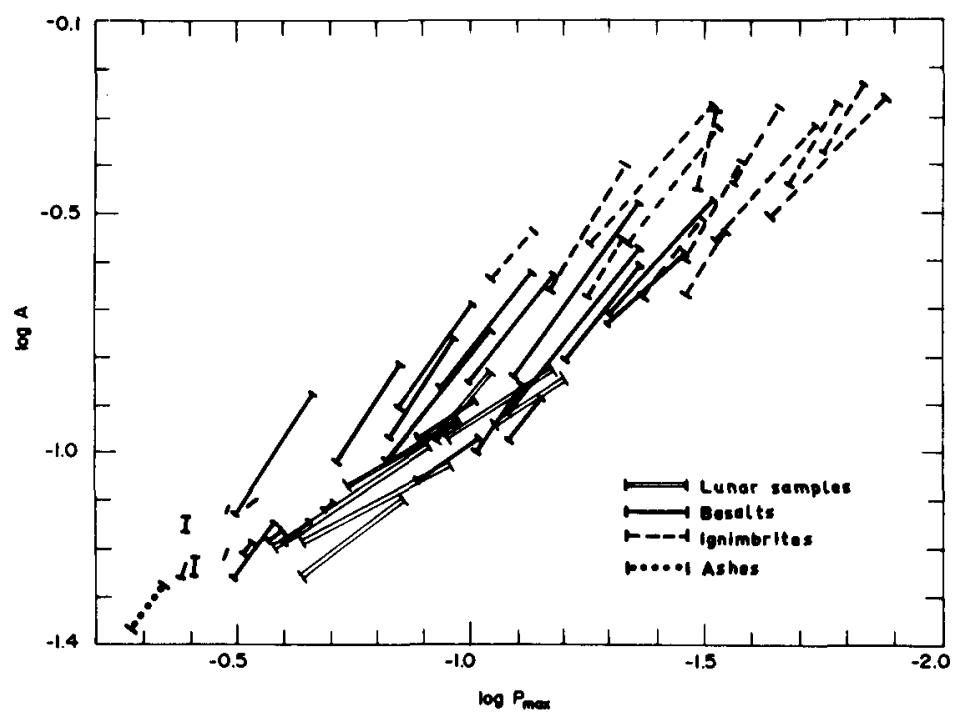

Figure 23. $-\log A$ versus $\log P_{\max }$ plot for 38 volcanic samples of pulverized basalts, ignimbrites, and ashes, and five Apollo lunar fines samples, measured at five wavelengths. The left and right ends of each segment correspond respectively to the wavelengths 354 and $580 \mathrm{~nm}$.

\section{REFERENCES}

Dollfus, A. 1955, Étude des Planètes par la Polarisation de la Lumière. Doctoral Thesis, Univ. of Paris. (1964, NASA TT F-188.)

Dollfus, A. 1956, Polarisation de la Lumière Renvoyée par les Corps Solides et les Nuages Naturels. Ann. Astrophys. 19, 83.

Dollfus, A. 1961, Polarization Studies of Planets. Planets and Satellites (ed., G. P. Kuiper), pp. 389-390. Univ. of Chicago Press. Chicago.

Dollfus, A. 1962, The Polarization of Moonlight. Physics and Astronomy of the Moon (ed., Z. Kopal), ch. 5. Academic Press, Inc. London.

Dollfus, A., and Bowell, E. 1971, Polarimetric Properties of the Lunar Surface and Its Interpretations, Part I. Telescopic Observations. Astron. Astrophys. 10, $29-53$.

Dollfus, A., Bowell, E., and Titulaer, C. 1971a, Polarimetric Properties of the Lunar Surface and Its Interpretations, Part II. Terrestrial Samples in Orange Light. Astron. Astrophys. 10, 450-466.

Dollfus, A., and Focas, J. 1969, La Planète Mars: La Nature de sa Surface et les Propriètés de son Atmosphere, d'Après la Polarisation de sa Lumière. I. Observations. Astron. Astrophys. 2, 63-74.

Dollfus, A., Focas, J., and Bowell, E. 1969, La Planète Mars: La Nature de sa Surface et les Propriètés de son Atmosphere, d'Après la Polarisation de sa Lumière. II. La Nature du Sol de la Planète Mars. Astron. Astrophys. 2, 105-121.

Dollfus, A., Geake, J., and Titulaer, C. 1971b, J. Geophys. Res., in press.

Dollfus, A., and Titulaer, C. 1971, Polarimetric Properties of the Lunar Surface and Its Interpretation. Part III. Astron. Astrophys., in press.

Geake, J., Dollfus, A., Garlick, G., Lamb, W., Walker, G., Steigmann, G., and Titulaer, C. 1970, Luminescence, Electron Paramagnetic Resonance and Optical Properties of 
Lunar Material From Apollo 11. Proc. Apollo 11 Lunar Sci. Conf. Geochim. Cosmochim. Acta 34, suppl. 1, vol. 3, 2127-2147.

Gehreis, T., Roemer, E., Taylor, R., and Zellner, B. 1970, Minor Planets and Related Objects. IV. Asteroid (1566) Icarus. Astron. J. 75, 186-195.

Lyot, B. 1929, Recherches sur la Polarisation de la Lumière des Planètes et de Quelques Substances Terrestres. Doctoral Thesis, Univ. of Paris. (1964, NASA TT F-187.)

Lyot, B. 1934, Polarisation des Petites Planètes. C.R. Acad. Sci. 199, 774.

McCord, B., Adams, J. B., and Johnson, T. V. 1970, Asteroid Vesta: Spectral Reflectivity and Compositional Implications. Science 168, 1445-1447.

Provin, S. 1955, Preliminary Observations of the Polarization of Asteroids. Publ. Astron. Soc. Pac. 67, 115.

Veverka, J. 1971, Photopolarimetric Observations of the Minor Planet Flora. Icarus 15(3), in press.

\section{DISCUSSION}

ARRHENIUS: For the case of Vesta, on the basis of the high albedo you rule out lunar dust and powder; probably you have in mind Apollo 11 or 12 dust because the new data on Apollo 14 indicate a much higher albedo.

DOLLFUS: We measured lunar fines on Apollo 12 on the lightest area available and none of them were higher than 0.25 .

ARRHENIUS: How about pure feldspar?

DOLLFUS: Pure feldspar is too transparent to produce the curve of polarization observed. It cannot be basalt because basalts are too absorbent.

ANDERS: I do not think we should dismiss the possibility of a regolith on asteroids. True, the escape velocity is low, but impact ejecta have a velocity distribution starting at zero, and so some fraction of the dust and debris will remain on the body. It does not matter that more material is lost than is gained in such an impact, because the loss is all in one place, the crater. The remainder of the body gets showered with low-velocity debris.

Moreover, a few percent of the stony meteorites in each class contain a noble-gas component that seems to represent trapped solar wind. Wänke suggested some years ago that implantation of solar-wind ions might happen in the regoliths of bodies without atmospheres, and this has been beautifully confirmed by Apollo 11 and 12 lunar soils and breccias. Now, meteorites apparently do not come from the Moon, and if the asteroids are too small to have a regolith, we will be hard pressed to find larger bodies without atmospheres where these gas-rich meteorites might form. Comets are too small and too far away and in a region where the solar-wind flux is very low. The moons of Jupiter are too large to permit escape of ejecta and cannot send material into terrestrial space with any efficiency. Thus we are left with the asteroids.

CHAPMAN: Anders has correctly noted that low escape velocity for asteroids need not imply that asteroids are not covered with a regolith or dust layer. The cratering and steady-state loss and accretion processes that govern the development of asteroidal regoliths are complex and depend on many factors. For example, in the case of a large population index for the impacting debris, a "sandblasting" effect may continually remove most or all of the regolith that otherwise would develop in the manner Anders mentioned.

Dr. Dollfus, in the final part of his presentation, has described how measurements of the maxima of asteroid polarization curves have some compositional implications. He argued that such measurements would be an important goal for a space mission. Ground-based spectral reflectivity studies are much cheaper, and they are far more diagnostic of mineralogical composition.

HAPKE: The relation between surface texture and the negative branch of polarization that Dr. Dollfus has described is certainly true. However, another property of materials that also affects the negative branch should be mentioned, and that is albedo. For a powder with a high albedo, such as pure feldspar or enstatite, the negative branch will be 
less pronounced than for a dark powder, such as Moon soil, so that there is some ambiguity in the interpretation of the negative polarization branch if the albedo is not known.

VEVERKA: I just want to note that the Lyot polarization curves for Ceres and Vesta were obtained by a rather insensitive photographic method and do not agree too well with more recent photoelectric measurements. One therefore should not base any inferences on them. 